\title{
iShim and Water Excitation Improves the Signal-to-Noise Ratio on q-Space Imaging: A Single-Center Clinical Study
}

\author{
Yoshifumi Sone', Junichi Hata $2,3,4$, Yasushi Sera ${ }^{5}$, Katsuya Maruyama6, Thomas Benkert7, \\ Zhouchen Lu', Jun Kasahara', Hideyuki Okano ${ }^{2,3,4}$, Morio Matsumoto ${ }^{8}$, Masaya Nakamura8, \\ Daisuke Nakashima ${ }^{8 *}$, Takeo Nagura ${ }^{8}$ \\ ${ }^{1}$ Medical Scanning Tokyo, Tokyo, Japan \\ ${ }^{2}$ Central Institute for Experimental Animals, Kanagawa, Japan \\ ${ }^{3}$ Department of Physiology, Keio University School of Medicine, Tokyo, Japan \\ ${ }^{4}$ Riken Brain Science Institute, Saitama, Japan \\ ${ }^{5}$ Institute for Integrated Sports Medicine, Keio University School of Medicine, Tokyo, Japan \\ ${ }^{6}$ MR Research \& Collaboration Department, Diagnostic Imaging Business Area, Siemens Healthcare K.K., Tokyo, Japan \\ ${ }^{7}$ Siemens Healthcare GmbH, Erlangen, Germany \\ ${ }^{8}$ Department of Orthopaedic Surgery, Keio University School of Medicine, Tokyo, Japan \\ Email: *nakashima@keio.jp
}

How to cite this paper: Sone, Y., Hata, J., Sera, Y., Maruyama, K., Benkert, T., Lu, Z., Kasahara, J., Okano, H., Matsumoto, M., Nakamura, M., Nakashima, D. and Nagura, T. (2018) iShim and Water Excitation Improves the Signal-to-Noise Ratio on q-Space Imaging: A Single-Center Clinical Study. Open Journal of Radiology, 8, 244-259. https://doi.org/10.4236/ojrad.2018.84028

Received: October 30, 2018

Accepted: November 20, 2018

Published: November 23, 2018

Copyright $\odot 2018$ by authors and Scientific Research Publishing Inc. This work is licensed under the Creative Commons Attribution International License (CC BY 4.0).

http://creativecommons.org/licenses/by/4.0/

\section{(c) (i) Open Access}

\begin{abstract}
Purpose: This study aimed to examine whether the signal-to-noise ratio (SNR) could be increased by combining integrated slice-by-slice shimming (iShim) with a fat suppression (FS) method other than short-tau inversion recovery (STIR) in diffusion-weighted imaging (DWI) and q-space imaging (qsi). Methods: We acquired DWI images (b-values: 0 and nine steps from 400 to $10,000 \mathrm{~s} / \mathrm{mm}^{2}$ for six axes) using a prototypical single-shot echo planar imaging sequence by combining two types of shimming (3D Shim and iShim) and two types of FS (STIR and water excitation [WE]) in 10 volunteers. In the DWI study, the SNR for each b-value, FS effect in the b0 image, and distortion in the added image ( $\mathrm{b} 0$ - b10,000) were evaluated for the above-mentioned four imaging methods. qsi involved original DWI images. In the qsi study, the SNR was evaluated. Results: With regard to both 3D Shim and iShim, the SNRs were significantly higher when using WE than when using STIR in b0 b900 images $(\mathrm{p}<0.01)$. For 3D Shim, however, the SNR increase effect of WE disappeared $(\mathrm{p}<0.01)$ at b1600 or higher. For iShim, the SNR increase effect of WE was maintained up to b3600 ( $p<0.01)$. The FS effect of STIR was superior to that of WE $(\mathrm{p}<0.01)$. Although WE had a weak FS effect, its FS effect was greater with iShim than with 3D Shim $(p<0.01)$. In added images, with iShim, the spinal cord area became significantly smaller $(\mathrm{p}<0.05)$, resulting
\end{abstract}


in a less distorted image when compared with the $3 \mathrm{D}$ shim image. On qsi, with regard to both 3D Shim and iShim, the SNRs were significantly higher when using WE than when using STIR (3D Shim: $p<0.05$; iShim: $p<0.01$ ). The SNR of iShim/WE was superior to that of 3D Shim/WE ( $p<0.05)$. Conclusion: The combination of iShim and WE has a high SNR on qsi.

\section{Keywords}

iShim, Fat Suppression, Water Excitation, Diffusion-Weighted Imaging, q-Space Imaging (qsi)

\section{Introduction}

The qualitative evaluation of the central nervous system (CNS) using magnetic resonance imaging (MRI) has recently become possible owing to the introduction of diffusion-weighted imaging (DWI). Diffusion tensor imaging (DTI) is a DWI approach incorporating the concept of anisotropy of diffusion restriction, and it was first developed in the CNS, especially the brain. The utility of the approach in the spinal cord, which is a part of the CNS, has been reported in animal experiments [1] [2] [3] and clinical trials [4] [5] [6] following evaluation in the brain. DTI parameters have been used to estimate the presence of a biological diffusion barrier (myelin) in the CNS [7] [8] [9]. DTI is based on the assumption that water molecules follow Gaussian distribution. In human tissues, however, water molecules diffuse according to a non-Gaussian distribution [10]. q-space imaging (qsi) [11] addresses this aspect. qsi is a DWI approach that considers non-Gaussian diffusion for the water molecule diffusion pattern in the human body, and it has shown contrast exceeding that with DTI in animals [12] [13]. Previously, qsi had a long acquisition time and was difficult to apply clinically. We recently successfully performed qsi in a clinical setting within an acceptable acquisition time ( $<8$ minutes) with reduction of the number of b-value steps and without loss of the characteristic probability density function (PDF) curve [13] [14]. qsi has been used for the evaluation of the brain in multiple sclerosis patients [14]. Similar use of qsi for the evaluation of the spinal cord, which shows common diseases related to demyelination, such as cervical myelopathy, is expected. However, as the spinal cord is smaller than the brain, an increase in the signal-to-noise ratio (SNR) is required for adaptation to the spinal cord in order to obtain images that are easy to understand visually.

Generally, on DWI, it is important to maintain a sufficient SNR in high-b-value images to achieve a high spatial resolution [15] [16]. Echo time (TE), and repetition time (TR) are representative parameters that affect the SNR. With regard to TE, a sufficiently long diffusion time is important for qsi. However, if the diffusion time is increased, TE increases. Similarly, with regard to TR, the SNR does not change with a certain time or a longer time. Therefore, it is difficult to improve the SNR only by adjusting TE and TR. In addition, as a chemical shift 
artifact (CSA) always occurs in DWI or echo planar imaging, it is indispensable to use fat suppression in combination. Studies have reported that the use of short-tau inversion recovery (STIR) in combination with DWI leads to an improvement in image quality [17] [18] [19]. However, STIR has the disadvantage of a lower SNR when compared to that for other fat suppression methods [15].

It has been reported that integrated slice-by-slice shimming (iShim) is associated with less distortion and results in an increased SNR in whole-body diffusion-weighted examinations, particularly in the cervical region [20] [21]. As mentioned above, it is difficult to improve the SNR with general parameters in DWI, and the SNR decreases with STIR combination. Thus, we examined whether the SNR could be increased by combining iShim with a fat suppression method other than STIR in DWI and qsi.

\section{Materials and Methods}

\subsection{Study Population}

This cross-sectional study included 10 Japanese non-symptomatic volunteers (mean [standard deviation (SD)] age, 33.5 [9.2] years; 8 males, 2 females), who were recruited from Keio University. We obtained informed consent from all volunteers. Nurick et al. [22] reported that cervical myelopathy develops at the age of over fifty years. For this reason, we selected healthy volunteers under the age of fifty this time. This study was approved by the ethics committee of Keio University School of Medicine (Approval No. 20170024). All participants provided informed consent to participate in the study.

\subsection{MRI Protocol}

All participants underwent imaging of the cervical-upper thoracic spine (i.e., occipital bone-the upper thoracic spine) using a 3T MRI scanner (MAGNETOM Skyrafit 3T, Siemens Healthcare, Erlangen, Germany) with a 64-channel head-neck coil (Siemens Healthcare). T2 weighted imaging and axial qsi was performed.

The imaging parameters for qsi using a prototypical spin-echo-type single-shot echo-planar DWI sequence were as follows: TR, 10,000 ms; TE, $137 \mathrm{~ms}$; average number, 2; field of view (FOV), $273 \times 273 \mathrm{~mm}^{2}$; matrix size, $256 \times 256$; resolution, $1.06 \times 1.06 \mathrm{~mm}^{2}$; slice thickness, $6 \mathrm{~mm}$; acceleration mode, generalized autocalibrating partial parallel acquisition; acceleration factor, 2; acquisition time, 9 min $56 \mathrm{~s}$. There were $10 \mathrm{~b}$-values $(0,400,900,1600,2500,3600,4900$, 6400,8100 , and $10,000 \mathrm{~s} / \mathrm{mm}^{2}$ ), and each b-value, except b0, had diffusion encoding in six directions. Data from the six directions were acquired separately and then averaged. The corresponding q-values for the $10 \mathrm{~b}$-values were $0,112.5$, $168.8,225.1,281.3,337.6,393.9,450.2,506.4$, and $562.7 \mathrm{~cm}^{-1}$, respectively. The gradient length $(\delta)$ and time between the two leading edges of the diffusion gradient $(\Delta)$ were 29.4 and $44.0 \mathrm{~ms}$, respectively. Four types of qsi images were obtained by combining two types of main magnetic field (B0) shim modes (conventional 3D Shim and iShim) and two types of fat suppression (water excitation 
[WE], $\delta 44.0 \mathrm{~ms}$ and $\Delta 66.9 \mathrm{~ms}$, and STIR, $\delta 41.2 \mathrm{~ms}$ and $\Delta 67.0 \mathrm{~ms}$ ). We evaluated the usefulness of iShim by examining the SNR and fat suppression effect among the aforementioned four types of qsi images.

\subsection{Shimming Techniques}

Conventional 3D Shim and iShim protocols were as previously described [21]. Briefly, for the conventional 3D Shim protocol, high-order shimming (up to the second order) was used for the entire slice stack of each bed position, and the acquisition of the field map was included in the automated scanner adjustment. For the iShim protocol, the acquisition of the field map was integrated into the prototypical single-shot DWI echo-planar imaging sequence. Using a double-echo gradient-echo scan, a 2D field map is acquired for each slice during an initial calibration phase, and slice-wise center frequencies and optional linear shim terms are calculated. Prior to the acquisition of each slice during the imaging phase, the frequency and the linear shim terms are dynamically updated. In contrast to this slice-wise, dynamic shimming approach, only a single center frequency and shim setting for the entire stack of slices are determined with conventional frequency adjustment and 3D shimming. Therefore, the target shim volume for iShim is much smaller which is why performance of shimming is improved. Acquisition time of the field map for iShim was approximately 540 milliseconds per slice, resulting in 19 seconds for a 35-slice station. Processing time of the field map was negligible.

\subsection{Fat Suppression Techniques}

Water excitation is a "spatial-spectral" pulse sequence designed to simultaneously select a spatial band (section thickness) and a spectral band (water) that excites the water inside a section and leaves the fat as well as tissues outside the section unaltered [23] [24]. STIR was introduced as a variant of previously used inversion-recovery sequences in low-field-strength MRI [25] [26].

\section{5. qsi}

qsi calculations were performed using an in-house program (developed in $\mathrm{C}++$; Embarcadero Technologies, Inc., Austin, TX, USA). The diffusion values and their calculation procedures were as previously described [27] [28] [29]. Briefly, the diffusion coefficient (D) and the signal decay of diffusion measurements were defined according to the following formula:

$$
\ln \left(S / S_{0}\right)=-\gamma^{2} G^{2} \delta^{2}(\Delta-\delta / 3) D=-b D
$$

where $S / S_{0}$ is the normalized diffusion signal, $\gamma$ is the proton gyromagnetic ratio (42.577 MHz/T), $G$ and $\delta$ are the amplitude and duration of motion-probing gradients (MPGs), respectively, and $\Delta$ is the separation time between the leading edges of these gradients. When normalization was performed for formula (1), the following formula is obtained: 


$$
E(q)=\exp \left(-4 \pi^{2} q^{2} d \Delta D\right)
$$

where $E(q)$ is the measured signal intensity decay as a function of $q$.

The non-Gaussian PDF of water diffusion was obtained with Fourier transformation of formula (2) according to the Stejskal-Tanner diffusion preparation [30]. After Fourier transformation of formula (2), the following formula is obtained:

$$
P(R, \Delta)=1 /\left((\sqrt{4 \pi D \Delta}) * \exp \left(-R^{2} / 4 D \Delta\right)\right)
$$

The PDF $[P(R, \Delta)]$ giving the probability density that a molecule moves the distance $\mathrm{R}$ during the time $\Delta$ is accordingly calculated [31]. If the object to be measured includes one element, the PDF shows Gaussian distribution. However, if the object includes several elements, the PDF shows non-Gaussian distribution, reflecting the complexity of the measured object. The important theory in qsi is that Fourier transformation of signal decay with respect to the b-value provides the PDF for diffusion by using multiple $q$-values. In this case, the q-value, which represents the horizontal axis, is defined by the following formula:

$$
q=\gamma \delta G / 2 \pi
$$

The figure of the calculated PDF can be characterized by full width at half maximum (FWHM) and Kurtosis. Kurtosis can be calculated using the following formula:

$$
\text { Kurtosis }=\frac{1}{N} \sum\left(\frac{x_{i}-\bar{x}}{S D}\right)^{4}-3
$$

where $N$ is the number of data points (i.e., number of b-value steps used), $\mathrm{x}$ is the probability value (a.u.) obtained from the PDF, and SD is the standard deviation of the $\mathrm{x}$ values.

\subsection{SNR Calculation}

The imaging values were measured using ImageJ $1.48 \mathrm{v}$ software (available at http://rsbweb.nih.gov/ij/; National Institutes of Health, Bethesda, MD, USA). The regions of interest (ROIs) were set on the C2/3 level spinal cord [32] (Figures 1(A)-(E)) and background (Figure 1(D)). In DWI analyses, which are associated with qsi, the value of the b0 image and the average value of the six directions in each of the other images (b400 to b10,000 images) were obtained. The mean signal intensity (SI) of the spinal cord and the SD of the SI at the background as noise were measured on DWI for the calculation of the SNR. On qsi, as background noise cannot be measured; we defined the SD of the SI the spinal cord as noise. The SNR of DWI and that of qsi were calculated using the following formulas:

$$
\mathrm{SNR} \text { of DWI }=\left(2-\frac{2}{\pi}\right)^{\frac{1}{2}} \frac{\mathrm{SI}_{\mathrm{SC}}}{\mathrm{SD}_{\mathrm{BG}}}
$$



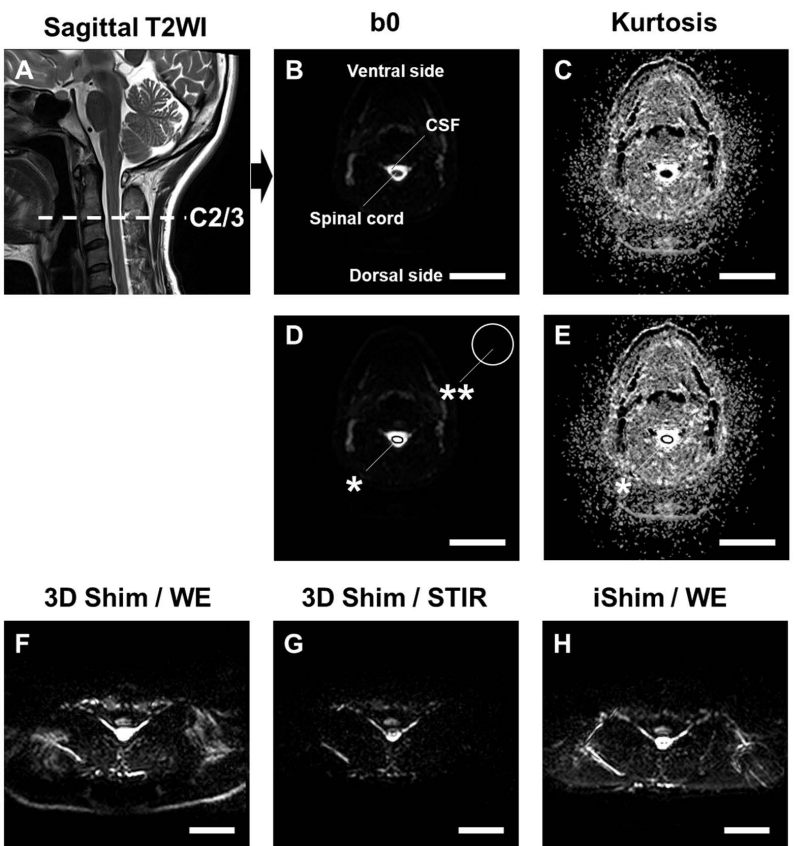

\section{iShim / WE}
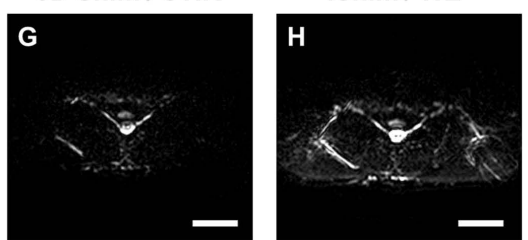

iShim / STIR
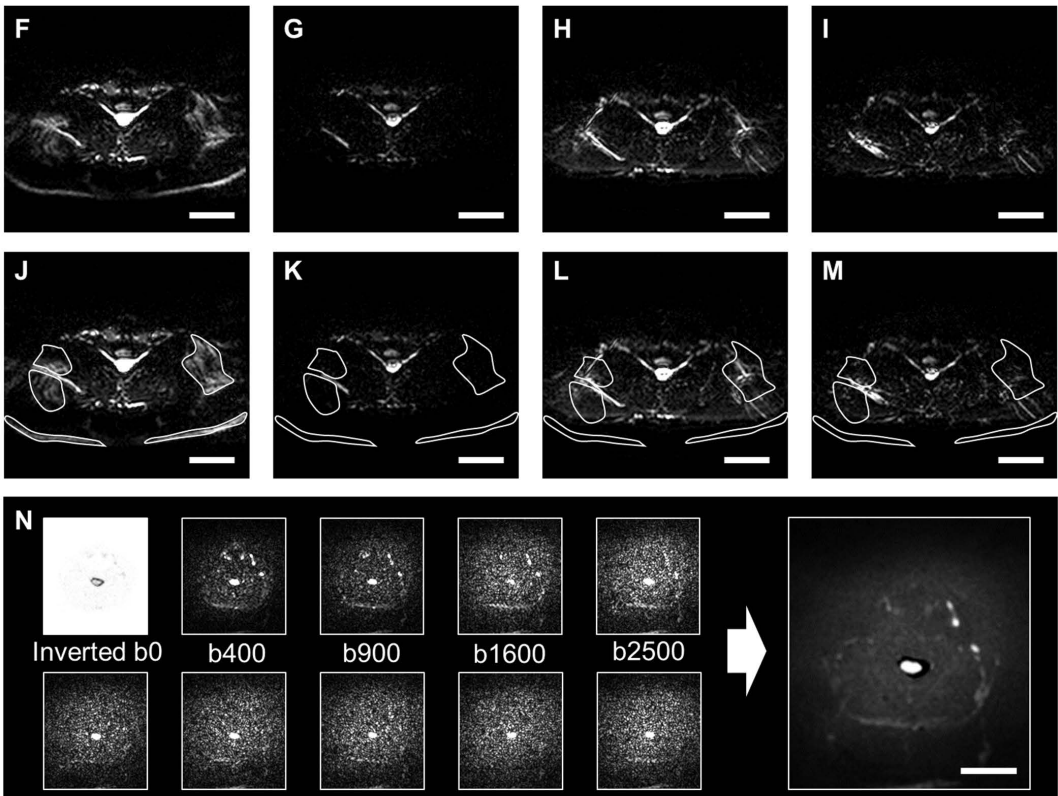

b3600
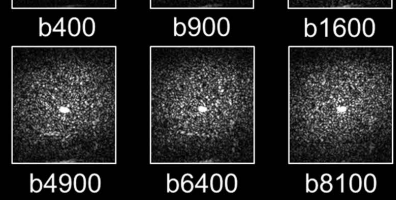

b10000

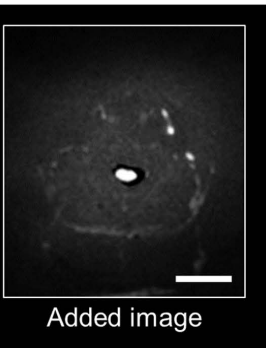

Figure 1. The setting of regions of interest (ROIs) in axial cervical spinal magnetic resonance images and the construction of images for the evaluation of distortion. (A) Sagittal T2 weighted image for reference. Axial C2/3 level DWI and qsi were used for analyses; (B) b0 reference image; (C) Kurtosis reference image; (D) Setting of a ROI at the spinal cord $\left(^{*}\right)$ and a $4 \mathrm{~cm} \times 4 \mathrm{~cm}$ ROI at the background $\left(^{* *}\right)$ in the b0 image (B); (E) Setting of a ROI at the spinal cord $\left(^{\star}\right)$ in the Kurtosis image (C); (F) 3D Shim/water excitation (WE) reference image at cervicothoracic junction level; (G) 3D Shim/short-tau inversion recovery (STIR) reference image at cervicothoracic junction level; $(\mathrm{H})$ iShim/WE reference image at cervicothoracic junction level; (I) iShim/STIR reference image at cervicothoracic junction level; (J, K, L, and M) Setting of ROIs at the parts where the fat suppression effect was insufficient in (F, G, H, and I); (N) Construction of images for the evaluation of distortion. An added image is constructed with the inverted b0 image and the images from b400 to b10,000; CSF: cerebrospinal fluid, Scale bar: $50 \mathrm{~mm}$.

$$
\mathrm{SNR} \text { of } \mathrm{qsi}=\frac{\mathrm{SI}_{\mathrm{sc}}}{\mathrm{SD}_{\mathrm{sc}}}
$$

where $\mathrm{SI}_{\mathrm{SC}}$ is the $\mathrm{SI}$ of the spinal cord, $\mathrm{SD}_{\mathrm{BG}}$ is the $\mathrm{SD}$ of the $\mathrm{SI}$ at the background, 
and $\mathrm{SD}_{\mathrm{SC}}$ is the $\mathrm{SD}$ of the $\mathrm{SI}$ at the spinal cord.

\subsection{Evaluation of the Fat Suppression Effect}

The fat suppression effect was evaluated using b0 images at cervicothoracic junction level (Figures $1(\mathrm{~F})$-(I)). First, we confirmed visually that the imaging method with the lowest fat suppression effect was 3D Shim/WE (Figure 1(F) and Figure $1(\mathrm{~J})$ ). To use the b0 image of 3D Shim/WE, we qualitatively set the ROIs on areas where fat suppression was insufficient (Figures $1(J)-(M)$ ). To use these ROIs, we compared the mean SI in each of the above-mentioned four types of b0 images (Figures $1(\mathrm{~F})-(\mathrm{M})$ ).

\subsection{Evaluation of Distortion}

Distortion was evaluated using the size of the spinal cord in the additionally calculated added image, which was generated as follows: First, the b0 image was inverted in order to display the spinal cord with a high SI when compared to that of the surrounding cerebrospinal fluid region. Afterwards, the inverted b0 image and the original b400 - b10,000 images of all axes were summed up. If the distortion is strong, the spinal cord regions do not overlap and are broadly displayed hyperintense in the added image (Figure $1(\mathrm{~N})$ ). To compare the size of the spinal cord in the added image among the above-mentioned four types of imaging methods, we evaluated the strength of distortion. Each value was measured by two experts (Y.S. and D.N. [13 and 11 years of experience, respectively]) with consensus. To assess the reproducibility of the calculations, blinded analyses were performed, and they involved independent triplicate analyses on different days. Reproducibility was quantified using the intraclass correlation coefficient (ICC) for absolute agreement. The ICC values were interpreted as follows: 0.81 - 1.0, substantial agreement; 0.61 - 0.80, moderate agreement; 0.41 - 0.60, fair agreement; $0.11-0.40$, slight agreement; $0.00-0.10$, virtually no agreement [33]. An ICC value $>0.81$ was considered to represent good agreement.

\subsection{Statistical Analysis}

Data are presented as mean \pm standard deviation. Multiple comparisons among the four imaging methods were performed using Student's $t$-tests with Bonferroni correction. All statistical analyses were performed using SPSS Statistics software, version 24 (IBM Corp., Armonk, NY, USA). The significance level for all tests was set at $\mathrm{p}<0.05$.

\section{Results}

\subsection{Calculation of Reproducibility}

Blinded assessments (setting ROIs) involving independent triplicate analyses were performed. The ICC for intraobserver reproducibility was 0.914 , indicating good reproducibility among the three analyses, and the ICC for interobserver reproducibility was 0.921 , indicating good reproducibility among the observers. 


\subsection{DWI Analysis}

Representative DWI images (b0, b900, b10,000, and added images for the above-mentioned four imaging methods) are presented in Figure 2.

\subsubsection{SNR}

The SNRs were higher when combining WE than when combining STIR in b0 images (Figures 2(A)-(D)). The SNRs are quantitatively presented in Figure 3. With regard to both 3D Shim and iShim, the SNRs were significantly higher when combining WE than when combining STIR in b0 - b900 images $(\mathrm{p}<0.01)$ (Figures 3(A)-(C)). However, for 3D Shim, the SNR increase effect of WE disappeared at b1600 or higher $(\mathrm{p}<0.01)$ (Figure $3(\mathrm{D}))$. Interestingly, for iShim, the SNR increase effect of WE was maintained up to b3600 ( $p<0.01)$ (Figures 3(E)-(G)). Table 1 summarizes the results of the SNR on DWI. At b6400 or higher, there was no difference in the SNR among the four imaging groups on one-way analysis of variance (Figures $3(\mathrm{H})-(\mathrm{J})$ and Table 1 ).

\subsubsection{Fat Suppression Effect}

As the b-value increased, the fat suppression effect of STIR, which is superior to

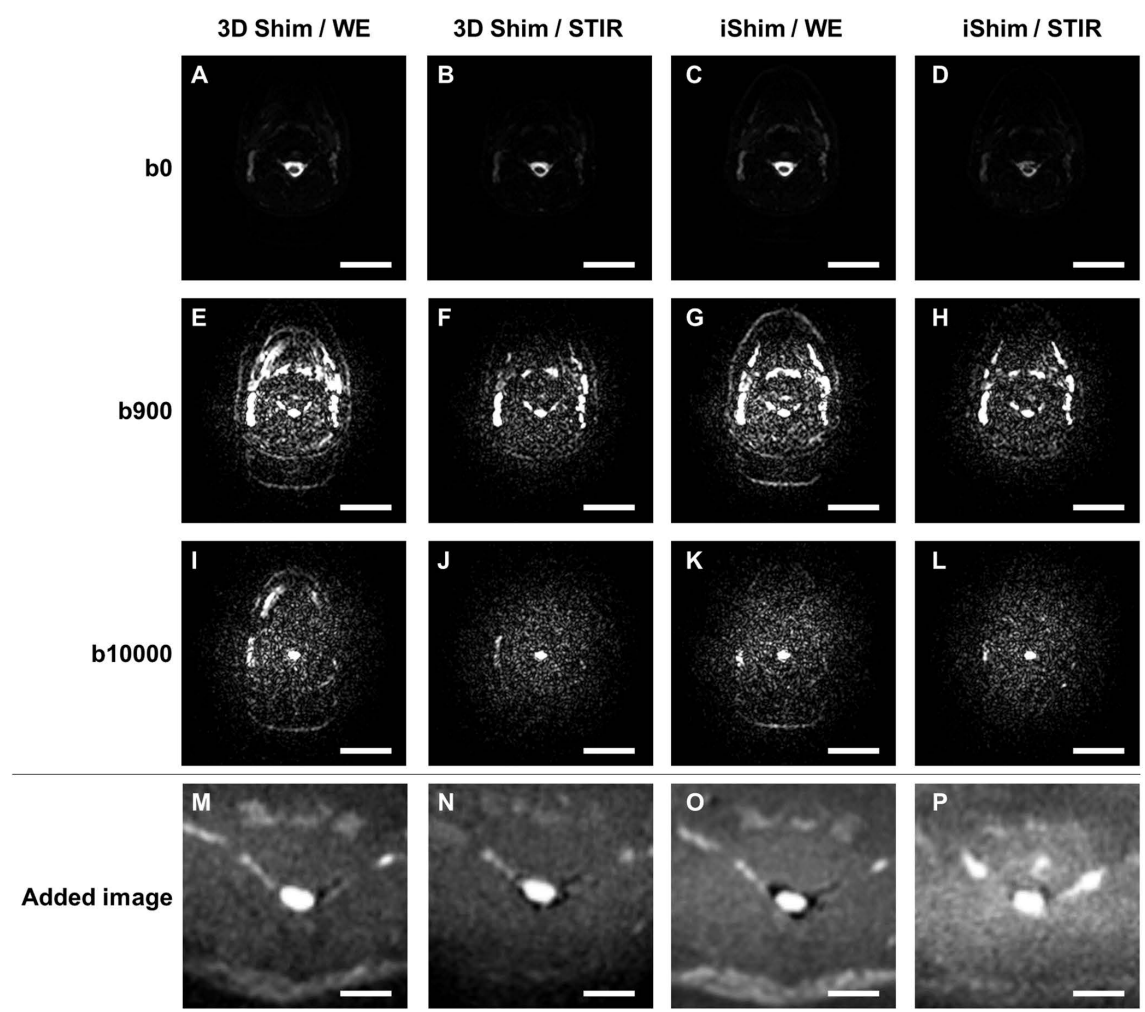

Figure 2. Representative diffusion-weighted images (b0, b900, b10,000, and added images) for the four imaging methods. Contrast is aligned for the b-value images (each type) and the added image. (A, E, I, and M) 3D Shim/water excitation (WE) images; (B, F, J, and N) 3D Shim/short-tau inversion recovery (STIR) images; (C, G, K, and O) iShim/WE images; (D, H, L, and P) iShim/STIR images. The first row presents b0 images, second row presents $\mathrm{b} 900$ images, third row presents b10,000 images, and fourth row presents added images. Scale bar: $50 \mathrm{~mm}(\mathrm{~A} \mathrm{-} \mathrm{L)}$ and $20 \mathrm{~mm}(\mathrm{M} \mathrm{-} \mathrm{P).}$ 

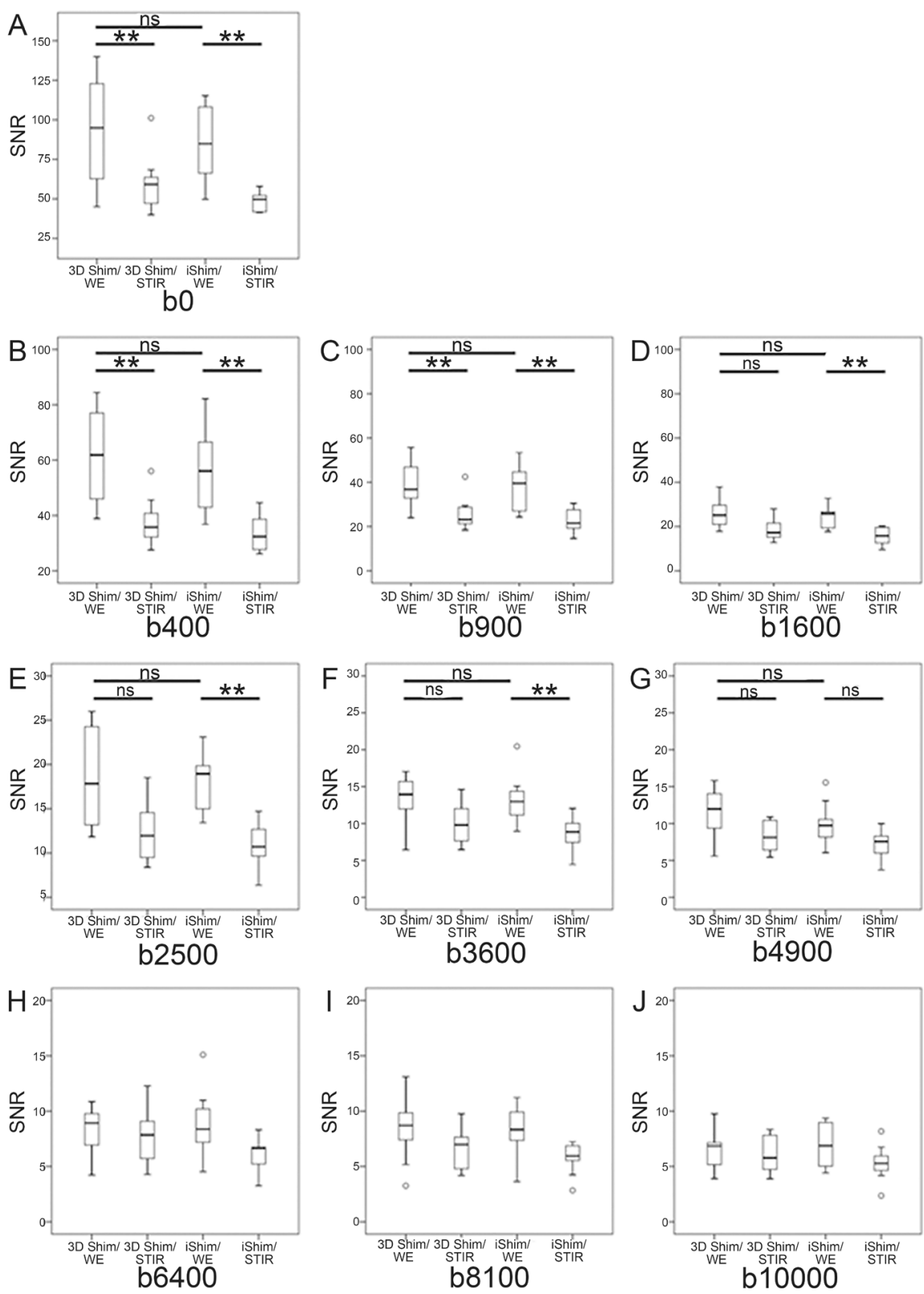

Figure 3. Quantification of the signal-to-noise ratio (SNR) on diffusion-weighted imaging (10 volunteers in each group). Box and whisker plots: the bottom and top of the box are the first and third quartiles, respectively, and the band inside the box is the second quartile (the median). The ends of the whiskers represent the minimum and maximum of the data. White circles represent outliers. Student's $t$-test with Bonferroni correction was used to evaluate the relationships among the four imaging groups for each b-value: b0 (A), b400 (B), b900 (C), b1600 (D), b2500 (E), b3600 (F), b4900 (G), b6400 (H), b8100 (I), and $b 10,000(\mathrm{~J})$. There is no difference at b6400 or higher on one-way analysis of variance. ns: not significant, ${ }^{* *}: \mathrm{p}<0.01$.

WE, became clearer (Figures 2(A)-(L)). Although WE has a weak fat suppression effect, its fat suppression effect is greater with iShim than with 3D Shim (Figures $2(\mathrm{E})-(\mathrm{L}))$. These findings were confirmed quantitatively $(\mathrm{p}<0.01)$ (Figure 4(A)). 
Table 1. Summary of the results of the signal-to-noise ratio in diffusion-weighed images and q-space imaging images (four imaging methods).

\begin{tabular}{cccccc}
\hline & 3D Shim/WE & 3D Shim/STIR & iShim/WE & iShim/STIR & $\mathrm{p}^{*}$ \\
\hline b0 & $94.41 \pm 32.11$ & $58.80 \pm 17.71$ & $86.31 \pm 22.77$ & $48.82 \pm 6.22$ & $\mathrm{p}<0.01$ \\
b400 & $62.05 \pm 16.28$ & $37.63 \pm 8.39$ & $55.97 \pm 15.29$ & $33.57 \pm 6.39$ & $\mathrm{p}<0.01$ \\
b900 & $39.23 \pm 9.58$ & $25.66 \pm 6.97$ & $37.46 \pm 10.15$ & $22.54 \pm 5.19$ & $\mathrm{p}<0.01$ \\
b1600 & $25.40 \pm 6.37$ & $18.87 \pm 5.49$ & $24.69 \pm 5.32$ & $15.79 \pm 3.84$ & $\mathrm{p}<0.01$ \\
b2500 & $18.25 \pm 5.47$ & $12.58 \pm 3.64$ & $17.98 \pm 3.15$ & $10.92 \pm 2.70$ & $\mathrm{p}<0.01$ \\
b3600 & $13.35 \pm 3.02$ & $10.14 \pm 2.86$ & $13.17 \pm 3.27$ & $8.74 \pm 2.32$ & $\mathrm{p}<0.01$ \\
b4900 & $11.38 \pm 3.39$ & $8.19 \pm 2.04$ & $9.98 \pm 2.73$ & $7.38 \pm 1.92$ & $\mathrm{p}<0.01$ \\
b6400 & $8.45 \pm 2.11$ & $7.84 \pm 2.81$ & $8.87 \pm 2.93$ & $6.24 \pm 1.56$ & $\mathrm{~ns}$ \\
b8100 & $8.35 \pm 2.69$ & $6.75 \pm 2.01$ & $8.05 \pm 2.32$ & $5.76 \pm 1.35$ & $\mathrm{~ns}$ \\
b10,000 & $6.53 \pm 1.86$ & $6.08 \pm 1.60$ & $6.92 \pm 1.88$ & $5.30 \pm 1.53$ & $\mathrm{~ns}$ \\
FWHM & $4.49 \pm 1.00$ & $3.86 \pm 0.49$ & $5.27 \pm 0.32$ & $3.49 \pm 0.68$ & $\mathrm{p}<0.01$ \\
Kurtosis & $3.96 \pm 0.69$ & $3.17 \pm 0.50$ & $4.78 \pm 0.72$ & $3.26 \pm 0.40$ & $\mathrm{p}<0.01$ \\
\hline
\end{tabular}

*analysis of variance, FWHM: full width at half maximum, WE: water excitation, STIR: short-tau inversion recovery, ns: not significant.

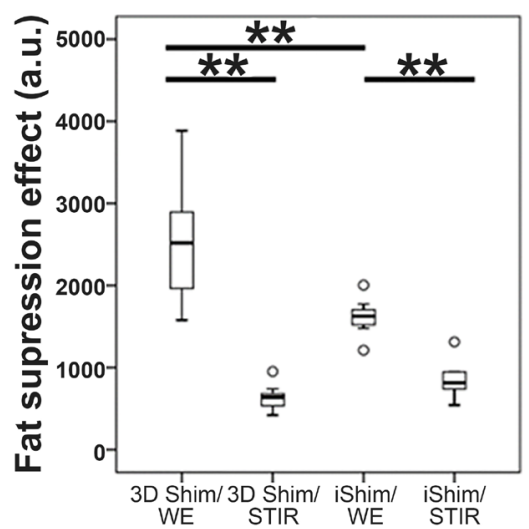

(A)

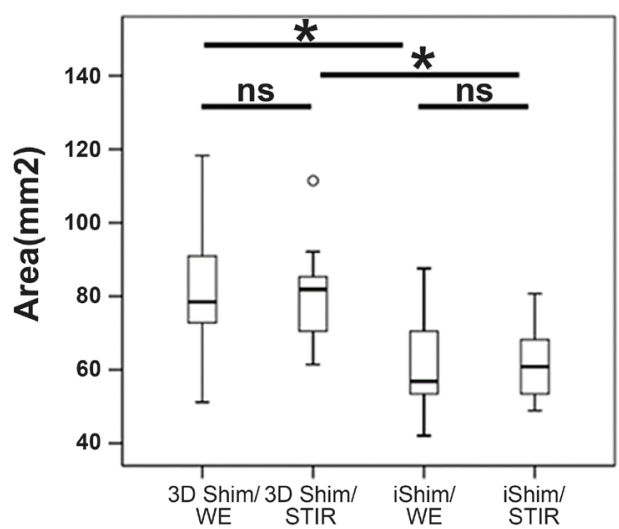

(B)

Figure 4. Quantification of the fat suppression effect in b0 images and the spinal cord area in added images (10 volunteers in each group). Box and whisker plots: the bottom and top of the box are the first and third quartiles, respectively, and the band inside the box is the second quartile (the median). The ends of the whiskers represent the minimum and maximum of the data. White circles represent outliers. Student's $t$-test with Bonferroni correction was used to evaluate the relationships among the four imaging groups for the fat suppression effect (A) and spinal cord area (B). a.u.: arbitrary unit, ns: not significant, ${ }^{*}: \mathrm{p}<0.05,{ }^{* *}: \mathrm{p}<0.01$.

\subsubsection{Evaluation of Distortion}

In the additionally calculated images, the spinal cord and cerebrospinal fluid region could be separated clearly by using iShim/WE (Figures 2(M)-(P)). In addition, by using iShim, the spinal cord area became significantly smaller $(\mathrm{p}<0.05)$, resulting in less distorted images when compared with the $3 \mathrm{D}$ Shim images (Figure 4(B)). Table 2 summarizes the results of the fat suppression effect and evaluation of distortion. 


\section{3. qsi Analysis}

FWHM and Kurtosis images for the above-mentioned four imaging methods are presented in Figure 5.

\section{$S N R$}

With regard to FWHM, the structure consisting of gray and white matter could be well visualized with iShim/WE (Figures 5(A)-(E)). With regard to Kurtosis, the SNR was high with the imaging method using WE (Figures 5(F)-(J)). The SNRs are quantitatively presented in Figure 6. With regard to both 3D Shim and iShim, the SNRs were significantly higher when using WE than when using STIR (3D Shim: $\mathrm{p}<0.05$; iShim: $\mathrm{p}<0.01$ ) (Figure $6(\mathrm{~A})$ and Figure 6(B)). However, unlike DWI, on qsi, the SNR of iShim/WE was superior to that of 3D Shim/WE $(\mathrm{p}<0.05)$. Table 2 summarizes the results of the SNR on qsi.

\section{Discussion}

We examined the appropriate shimming technique and combined fat suppression method for qsi, which involved various b-values from DWI. iShim/3D Shim

Table 2. Summary of the results of fat suppression insufficiency in b0 images and the spinal cord area in added images (four imaging methods).

\begin{tabular}{lccccc}
\hline & 3D Shim/WE & 3D Shim/STIR & iShim/WE & iShim/STIR & $\mathrm{p}^{*}$ \\
\hline $\begin{array}{l}\text { Fat suppression } \\
\text { effect (a.u.) }\end{array}$ & $2543.73 \pm 686.82$ & $637.39 \pm 145.47$ & $1623.83 \pm 205.25$ & $842.60 \pm 205.09$ & $\mathrm{p}<0.01$ \\
\begin{tabular}{c} 
Area $\left(\mathrm{mm}^{2}\right)$ \\
\hline
\end{tabular} & $61.72 \pm 9.50$ & $60.17 \pm 12.57$ & $79.91 \pm 13.92$ & $79.81 \pm 17.97$ & $\mathrm{p}<0.01$ \\
\hline
\end{tabular}

*analysis of variance, a.u.: arbitrary unit, WE: water excitation, STIR: short-tau inversion recovery.

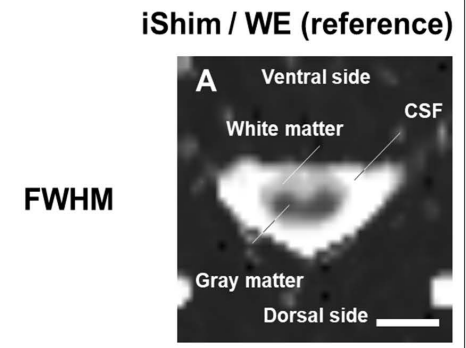

\section{D Shim / WE}
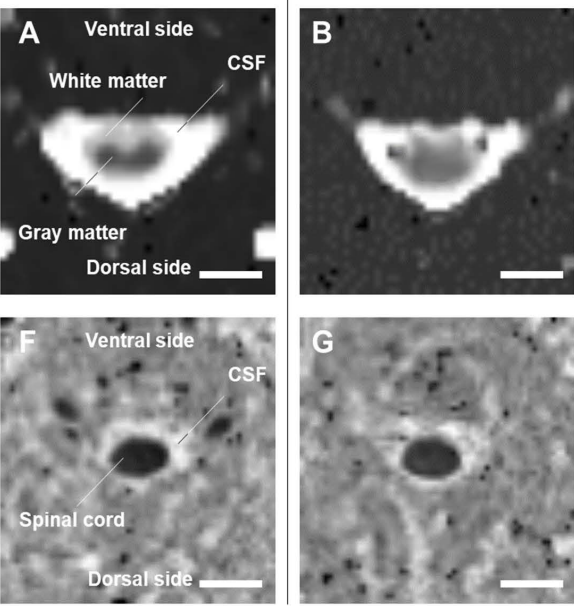

3D Shim / STIR
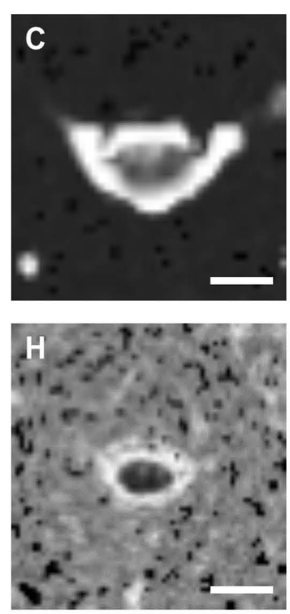

iShim / WE

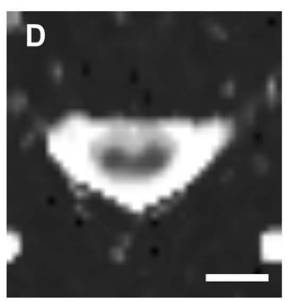

iShim / STIR
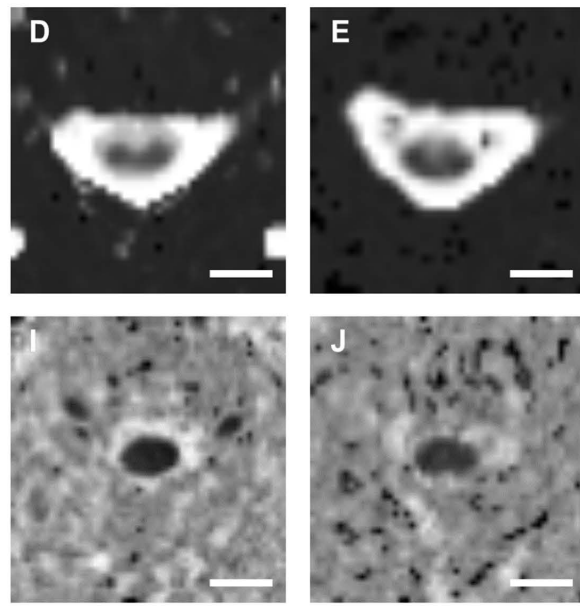

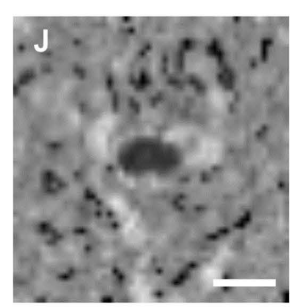

Figure 5. Representative q-space imaging images (full width at half maximum and Kurtosis) for the four imaging methods. Contrast is aligned for each qsi parameter. (A, F) iShim/water excitation (WE) reference images; (B, G) 3D Shim/WE images; (C, H) 3D Shim/short-tau inversion recovery (STIR) images; (D, I) iShim/WE images; (E, J) iShim/STIR images; The upper row presents full width at half maximum (FWHM) images, and the lower row presents Kurtosis images. CSF: cerebrospinal fluid, Scale bar: $10 \mathrm{~mm}$. 


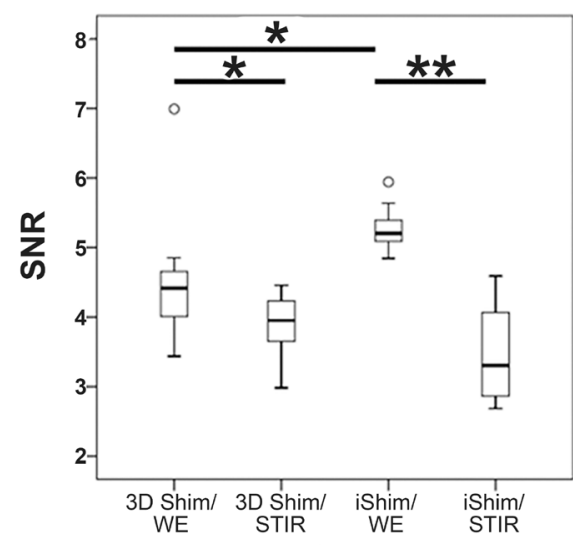

FWHM

(A)

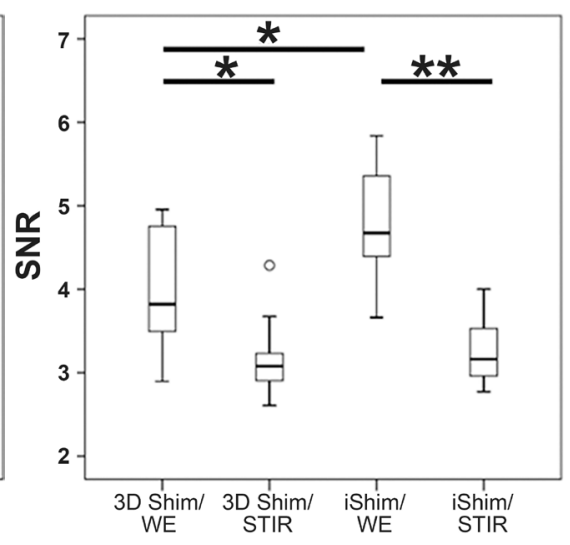

Kurtosis

(B)

Figure 6. Quantification of the signal-to-noise ratio (SNR) on q-space imaging (10 volunteers in each group). Box and whisker plots: the bottom and top of the box are the first and third quartiles, respectively, and the band inside the box is the second quartile (the median). The ends of the whiskers represent the minimum and maximum of the data. White circles represent outliers. Student's $t$-test with Bonferroni correction was used to evaluate the relationships among the four imaging groups for full width at half maximum (FWHM) (A) and Kurtosis (B). ${ }^{*}: \mathrm{p}<0.05,{ }^{* *}: \mathrm{p}<0.01$.

and STIR/WE DWI acquisitions with the exact same scan parameters were performed sequentially, and the impact of different shimming and fat suppression techniques on image quality was quantitatively examined.

In the DWI study, the SNR of WE was higher than that of STIR with both 3D Shim and iShim at a low b-value. STIR is an approach that utilizes the difference between the relaxation time of water and fat through the application of an IR pulse [25] [26]. The water signal, which is inverted by the inversion pulse, is not fully recovered at excitation. Therefore, longitudinal magnetization also decreases, resulting in a decrease in the SI of the spinal cord when compared with the finding using chemical-shift-based fat suppression methods. However, chemical-shift-based fat suppression techniques are very sensitive to B0 inhomogeneity, and thus, the image quality might be degraded by fat ghosting. As iShim helps to significantly reduce B0 inhomogeneity, chemical-shift-based fat suppression techniques might provide efficient fat suppression. Chemical-shiftbased fat suppression techniques, such as spectral adiabatic inversion recovery, are not compatible with iShim, as they exploit nonselective radio-frequency pulses. As an alternative, WE, which involves a binominal pulse, could be used with iShim [23] [24], because it is a slice-selective method. Therefore, it is possible to achieve selective excitation only when the longitudinal magnetization of fat is in equilibrium, using the difference between the frequencies of water and fat. Thus, it is considered that there is no decrease in the SI of the spinal cord and that the SNR increases when compared with images using STIR [23]. In addition, the SNR was maintained at a high b-value using iShim instead of 3D Shim. In order to further assess this phenomenon, a feature study of iShim was performed. 
On assessing distortion, it was found that distortion tended to be lower with iShim than with 3D Shim, irrespective of the use of WE or STIR. As 3D Shim measures the imaging region at the same time and determines the center frequency, a homogeneous magnetic field cannot be obtained in the cervical region with its complicated anatomical shape. On the other hand, iShim makes it possible to obtain a homogeneous magnetic field by measuring the B0 field map for each slice and performing slice-specific adjustments [21]. The neck region showed larger variations in slice to slice center frequency offset and linear frequency shift in the phase-encoding direction when compared with the results in other body regions. This explains why 3D Shim, which delivers a single-center frequency and a set of shim currents per station, is not reliable in the neck region [20].

In the qsi study, with regard to the final calculated image, WE maintained a higher SNR when compared with the SNR using STIR, and this was further reinforced by combining iShim. This is considered to be associated with the high SNR of WE, low distortion of iShim, and strong fat suppression effect of iShim in the original image. We showed that a high SNR could be obtained by using iShim together with WE for the final qsi image instead of the original image. Our findings might lead to the advancement of qsi analysis.

The present study has certain limitations. First, we could not implement fat suppression methods other than STIR and WE as iShim is not compatible with chemical shift selective imaging. Even with 3D Shim, a sufficient SNR might be obtained by combining other fat suppression methods. Second, participants in this study were healthy volunteers. Thus, the use of qsi for compression myelopathy and other such diseases is unknown. Imaging studies involving approaches with a sufficient SNR, such as iShim/WE, should be performed in patients in the future. Third, we only identified distortions between different b-values (e.g., due to eddy currents). However, distortions due to susceptibility, which are identical for all b-values, were not be identified. It is desirable to develop a quantitative distortion evaluation method in the future.

\section{Conclusion}

The combination of iShim and WE has a high SNR on qsi. iShim might show improved clinical performance with regard to the detection of suspicious lesions in the neck region.

\section{Acknowledgements}

The authors acknowledge the staff of META corporation and Medical Scanning Tokyo for MRI technological assistance.

\section{Disclosure Statement}

Hideyuki Okano is a paid scientific advisory board member of SanBio Co. Ltd. Thomas Benkert and Katsuya Maruyama are employees of Siemens Healthcare 
$\mathrm{GmbH}$, who provided crucial technical support with the MR sequence but were not involved in data acquisition and analysis. No conflicts of interest are declared for the remaining authors.

\section{Conflicts of Interest}

The authors declare no conflicts of interest regarding the publication of this paper.

\section{References}

[1] Fujiyoshi, K., Yamada, M., Nakamura, M., et al. (2007) In Vivo Tracing of Neural Tracts in the Intact and Injured Spinal Cord of Marmosets by Diffusion Tensor Tractography. Journal of Neuroscience, 27, 11991-11998. https://doi.org/10.1523/JNEUROSCI.3354-07.2007

[2] Takano, M., Hikishima, K., Fujiyoshi, K., et al. (2012) MRI Characterization of Paranodal Junction Failure and Related Spinal Cord Changes in Mice. PloS One, 7, e52904. https://doi.org/10.1371/journal.pone.0052904

[3] Konomi, T., Fujiyoshi, K., Hikishima, K., et al. (2012) Conditions for Quantitative Evaluation of Injured Spinal Cord by in Vivo Diffusion Tensor Imaging and Tractography: Preclinical Longitudinal Study in Common Marmosets. Neuroimage, 63, 1841-1853. https://doi.org/10.1016/j.neuroimage.2012.08.040

[4] Nakamura, M., Fujiyoshi, K., Tsuji, O., et al. (2012) Clinical Significance of Diffusion Tensor Tractography as a Predictor of Functional Recovery after Laminoplasty in Patients with Cervical Compressive Myelopathy. Journal of Neurosurgery. Spine, 17, 147-152. https://doi.org/10.3171/2012.5.SPINE1196

[5] Xiangshui, M., Xiangjun, C., Xiaoming, Z., et al. (2010) 3 T Magnetic Resonance Diffusion Tensor Imaging and Fibre Tracking in Cervical Myelopathy. Clinical Radiology, 65, 465-473. https://doi.org/10.1016/j.crad.2010.01.019

[6] Guan, X., Fan, G., Wu, X., et al. (2015) Diffusion Tensor Imaging Studies of Cervical Spondylotic Myelopathy: A Systemic Review and Meta-Analysis. PloS One, 10, e0117707. https://doi.org/10.1371/journal.pone.0117707

[7] Engelbrecht, V., Scherer, A., Rassek, M., Witsack, H.J. and Modder, U. (2002) Diffusion-Weighted MR Imaging in the Brain in Children: Findings in the Normal Brain and in the Brain with White Matter Diseases. Radiology, 222, 410-418. https://doi.org/10.1148/radiol.2222010492

[8] Pfefferbaum, A., Sullivan, E.V., Hedehus, M., Lim, K.O., Adalsteinsson, E. and Moseley, M. (2000) Age-Related Decline in Brain White Matter Anisotropy Measured with Spatially Corrected Echo-Planar Diffusion Tensor Imaging. Magnetic Resonance in Medicine, 44, 259-268. https://doi.org/10.1002/1522-2594(200008)44:2<259::AID-MRM13>3.0.CO;2-6

[9] Avram, A.V., Guidon, A. and Song, A.W. (2010) Myelin Water Weighted Diffusion Tensor Imaging. Neuroimage, 53, 132-138. https://doi.org/10.1016/j.neuroimage.2010.06.019

[10] Katsura, M., Suzuki, Y., Hata, J., et al. (2014) Non-Gaussian Diffusion-Weighted Imaging for Assessing Diurnal Changes in Intervertebral Disc Microstructure. Journal of Magnetic Resonance Imaging, 40, 1208-1214. https://doi.org/10.1002/jmri.24459

[11] Callaghan, P.T., Coy, A., MacGowan, D., Packer, K.J. and Zelaya, F.O. (1991) Diffraction-Like Effects in NMR Diffusion Studies of Fluids in Porous Solids. Nature, 351, 467-469. https://doi.org/10.1038/351467a0 
[12] Assaf, Y., Mayk, A. and Cohen, Y. (2000) Displacement Imaging of Spinal Cord Using q-Space Diffusion-Weighted MRI. Magnetic Resonance in Medicine, 44, 713-722. https://doi.org/10.1002/1522-2594(200011)44:5<713::AID-MRM9>3.0.CO;2-6

[13] Fujiyoshi, K., Hikishima, K., Nakahara, J., et al. (2016) Application of q-Space Diffusion MRI for the Visualization of White Matter. Journal of Neuroscience, 36, 2796-2808. https://doi.org/10.1523/JNEUROSCI.1770-15.2016

[14] Tanikawa, M., Nakahara, J., Hata, J., et al. (2017) q-Space Myelin Map Imaging for Longitudinal Analysis of Demyelination and Remyelination in Multiple Sclerosis Patients Treated with Fingolimod: A Preliminary Study. Journal of the Neurological Sciences, 373, 352-357. https://doi.org/10.1016/j.jns.2017.01.009

[15] Baron, P., Dorrius, M.D., Kappert, P., Oudkerk, M. and Sijens, P.E. (2010) Diffusion-Weighted Imaging of Normal Fibroglandular Breast Tissue: Influence of Microperfusion and Fat Suppression Technique on the Apparent Diffusion Coefficient. NMR in Biomedicine, 23, 399-405. https://doi.org/10.1002/nbm.1475

[16] Seo, Y., Wang, Z.J., Morriss, M.C. and Rollins, N.K. (2012) Minimum SNR and Acquisition for Bias-Free Estimation of Fractional Anisotropy in Diffusion Tensor Imaging-A Comparison of Two Analytical Techniques and Field Strengths. Magnetic Resonance Imaging, 30, 1123-1133. https://doi.org/10.1016/j.mri.2012.04.015

[17] Takahara, T., Imai, Y., Yamashita, T., Yasuda, S., Nasu, S. and Van Cauteren, M. (2004) Diffusion Weighted Whole Body Imaging with Background Body Signal Suppression (DWIBS): Technical Improvement Using Free Breathing, STIR and High Resolution 3D Display. Radiation Medicine, 22, 275-282.

[18] Kazama, T., Nasu, K., Kuroki, Y., Nawano, S. and Ito, H. (2009) Comparison of Diffusion-Weighted Images Using Short Inversion Time Inversion Recovery or Chemical Shift Selective Pulse as Fat Suppression in Patients with Breast Cancer. Japanese Journal of Radiology, 27, 163-167. https://doi.org/10.1007/s11604-009-0314-7

[19] Maehara, M., Ikeda, K., Kurokawa, H., et al. (2014) Diffusion-Weighted Echo-Planar Imaging of the Head and Neck Using 3-T MRI: Investigation into the Usefulness of Liquid Perfluorocarbon Pads and Choice of Optimal Fat Suppression Method. Magnetic Resonance Imaging, 32, 440-445. https://doi.org/10.1016/j.mri.2014.01.011

[20] Stemmer, A. and Kiefer, B. (2015) Combination of Integrated Slice-Specific Dynamic Shimming and Pixel-Wise Unwarping of Residual EPI Distortions. Proceedings of the International Society for Magnetic Resonance in Medicine, Toronto, 30 May-5 June 2015, p. 3729.

[21] Zhang, H., Xue, H., Alto, S., et al. (2016) Integrated Shimming Improves Lesion Detection in Whole-Body Diffusion-Weighted Examinations of Patients with Plasma Disorder at 3 T. Investigative Radiology, 51, 297-305.

[22] Nurick, S. (1972) The Natural History and the Results of Surgical Treatment of the Spinal Cord Disorder Associated with Cervical Spondylosis. Brain, 95, 101-108. https://doi.org/10.1093/brain/95.1.101

[23] Meyer, C.H., Pauly, J.M., Macovskiand, A. and Nishimura, D.G. (1990) Simultaneous Spatial and Spectral Selective Excitation. Magnetic Resonance in Medicine, 15, 287-304. https://doi.org/10.1002/mrm.1910150211

[24] Hauger, O., Dumont, E., Chateil, J.F., Moinard, M. and Diard, F (2002) Water Excitation as an Alternative to Fat Saturation in MR Imaging: Preliminary Results in Musculoskeletal Imaging. Radiology, 224, 657-663. 
https://doi.org/10.1148/radiol.2243011227

[25] Bydder, G. and Young, I. (1985) Clinical Use of the Partial Saturation and Saturation Recovery Sequences in MR Imaging. Journal of Computer Assisted Tomography, 9, 1020-1032. https://doi.org/10.1097/00004728-198511000-00004

[26] Bydder, G.M., Pennock, J., Steiner, R., Khenia, S., Payne, J. and Young, I.R. (1985) The Short TI Inversion Recovery Sequence-An Approach to MR Imaging of the Abdomen. Magnetic Resonance Imaging, 3, 251-254. https://doi.org/10.1016/0730-725X(85)90354-6

[27] Assaf, Y., Ben-Bashat, D., Chapman, J., et al. (2002) High b-Value q-Space Analyzed Diffusion-Weighted MRI: Application to Multiple Sclerosis. Magnetic Resonance in Medicine, 47, 115-126. https://doi.org/10.1002/mrm.10040

[28] Farrell, J.A., Smith, S.A., Gordon-Lipkin, E.M., Reich, D.S., Calabresi, P.A. and van Zijl, P.C. (2008) High b-Value q-Space Diffusion-Weighted MRI of the Human Cervical Spinal Cord in Vivo: Feasibility and Application to Multiple Sclerosis. Magnetic Resonance in Medicine, 59, 1079-1089. https://doi.org/10.1002/mrm.21563

[29] Jensen, J.H., Helpern, J.A., Ramani, A., Lu, H. and Kaczynski, K. (2005) Diffusional Kurtosis Imaging: The Quantification of Non-Gaussian Water Diffusion by Means of Magnetic Resonance Imaging. Magnetic Resonance in Medicine, 53, 1432-1440. https://doi.org/10.1002/mrm.20508

[30] Stejskal, E.O. and Tanner, J.E. (1965) Spin Diffusion Measurements: Spin Echoes in the Presence of a Time-Dependent Field Gradient. The Journal of Chemical Physics, 42, 288-292. https://doi.org/10.1063/1.1695690

[31] Ibrahim, T.S., Lee, R., Abduljalil, A.M., Baertlein, B.A. and Robitaille, P.M. (2001) Dielectric Resonances and B(1) Field Inhomogeneity in UHFMRI: Computational Analysis and Experimental Findings. Magnetic Resonance Imaging, 19, 219-226. https://doi.org/10.1016/S0730-725X(01)00300-9

[32] Urakawa, T., Matsuzawa, H., Suzuki, Y., Endo, N., Kwee, I.L. and Nakada, T. (2011) Analysis of Ascending Spinal Tract Degeneration in Cervical Spondylotic Myelopathy Using 3D Anisotropy Contrast Single-Shot Echo Planar Imaging on a 3.0-T System. Journal of Neurosurgery. Spine, 15, 648-653. https://doi.org/10.3171/2011.7.SPINE10843

[33] Shrout, P.E. (1998) Measurement Reliability and Agreement in Psychiatry. Statistical Methods in Medical Research, 7, 301-317. https://doi.org/10.1177/096228029800700306 